\title{
SIMPLE AND INSENSITIVE BOUNDS FOR A GRADING AND AN OVERFLOW MODEL
}

\author{
Nico M. van DIJK \\ Department of Applied Mathematics, Twente University of Technology, P.O. Box 217, 7500 AE Enschede, The Netherlands
}

Received November 1985

Revised February 1987

\begin{abstract}
Simple and intuitively obvious lower and upper bounds are suggested for a specific grading and an overflow model. The bounds are based on product-type modifications and are insensitive. Numerical support indicates a potential usefulness for quick engineering purposes.
\end{abstract}

queues * overflow * loss probabiiity * bounds * job-local-balance * insensitivity

\section{Introduction}

This paper presents computationally attractive and insensitive bounds for a special simple grading and an overflow model of practical interest. Beyond the results themselves the motivation for this paper is twofold:

(i) It further explores and supports a bounding methodology introduced in [3]. In this reference quick bounds were provided for finite single-server tandem queues. Numerical results indicated a potential practical usefulness of the bounds as fast reasonable indicators of the performance of the system. vith similar success the methodology was extended in [4] and [5] to finite multi-server tandem queues and first-in first-out delay systems. This paper shows that the same methodology also works quite well in some overflow situations.

(ii) It also addresses to a question raised in [1] and [9] for the gradir: 6 studied. This grading is not of product form nor insensitive. That is, its stationary distribution depends upon the service distributions by their distributional forms and not only their means. Yet, numerical results showed that it was 'nearly insensitive' for two-phase service distributions. The question thus arose up to what extent the system is sensitive and whether some measure of sensitivity can be secured. Such a question is of rather general interest and is responded by an insensitive lower and upper bound.
The bounding methodology summarizes as: 'Modify the original system in such a way that (i) the notion of job-local-balance is guaranteed, and

(ii) bounds for a performance measure of interest are expected.'

Herein, the notion of job-local-balance (JLB) is introduced in [6] and shown to be responsible for insensitive and product-type stationary distributions [6,7]. Roughly, it reads as:

'The rate out of a state due to any particular job equals the rate into that state due to that particular job.'

JLB-modifications can be found by first identifying the cause(s) for the JLB-failure and next to modify the queueing protocol in vicw of this (these) cause(s) as to repair JLB (physical approach). Results from [6, Sections 4, 5 and 6] can hereby be helpful. Another approach, which is currently investigated and applied to the same grading in [8] and [10], is to modify the transition diagram by deleting and adding arcs (graphical approach). Various modifications may hereby be found easier. Since, however, the physical approach is much more intuitively supported, it seems more appealing for quick engineering purposes and complex systems. This paper therefore follows the physical approach. The recognition of JLB-modifications which lead to bounds is mainly based on physical insight of the system. 
First, in Section 2, insensitive bounds will be established for the simple grading studied in [9]. The bounds are intuitively obvious. A formal proof will not be given but can be concluded from [8] or [2] for special situations. The lower bounds are chosen rather naively and in fact can be slightly improved as in [8]. The upper bounds had to be defined more artificially based on JLB. Numerical comparison illustrates that the bounds are quite reasonable indicators.

Next, in Section 3, insensitive and computationally attractive bounds are also obtained for a more complex overflow model which is of practical importance. A formal and general proof of these bounds is provided in [2]. In this case, also the lower bounds had to be defined more artificially based on JLB. Numerical results at least appear to be rather accurate for small systems and promising for further application.

\section{A simple grading and bounds}

This section concerns the asymmetric grading studied in [9]. This grading consists of 2 servers with a Poisson arrival stream 1 at server 1 and 2 at server 2 , both with parameter $\lambda$. A service request of stream 2 is lost if server 2 is busy. A service request of stream 1 , however, is first placed at server 1 but rerouted (overflowed) to server 2 if server 1 is busy. Only if both servers 1 and 2 are busy, a type 1 service request is lost. The service distribution function $G$ is assumed to be the same for both strean 1 and stream 2 at server 1 and 2 and to have a mean $\mu^{-1}$. We write $\rho=\lambda / \mu$ and are interested in the loss probability (call congestion) $B^{i}(G)$ for a service request of type $i=1$ and $i=2$.

The present system has no product form and fails insensitivity. A simple way of arguing the latter via JLB is as follows. Let $(i, j)$ denote the state in which a job of type $i$ and $j$ is present at server 1 and 2, respectively, where $i=0$ and $j=0$ denote that there is actually no job at that server. Thus, state $(0,1)$ denotes that there is no job at server 1 but a type-1 job at server 2 . Now recall the job-local-balance interpretation under the assumption of exponential services. Then the 'rate out of state $(0,1)$ due to that type-1 job equals $p(0,1) \mu$ with $p(0,1)$ the stationary probability of state $(0,1)$. The 'rate into' that state $(0,1)$ due to that type-1 job, however, is equal to 0 , since a type-1 arrival in state $(0,0)$ would lead to state $(1,0)$. Consequently, in this state job-local-balance fails. From [7] we may then conclude that insensitivity does not hold.

We wish to establish a lower and upper bound $B_{\mathrm{L}}^{i}$ and $B_{\mathrm{U}}^{i}$ of $B^{i}(G)$ independently of $G$ and for $i=1,2$. Lower bounds are naively found by simply disregarding the interconnection of the servers and assuming full availability of both servers to stream 1. With Erlang's loss formula this leads to

$B_{L}^{1}=\left[\rho^{2} / 2\right] /\left[\rho^{2} / 2+\rho+1\right] \leq B^{1}(G)$,

$B_{\mathrm{L}}^{2}=\rho /[\rho+1] \leq B^{2}(G)$.

Upper bounds, however, have to be found more artificially. In view of the failure of JLB in state $(0,1)$, we suggest the following modification. A job of type 1 at server 2 has to restart a complete new service at server 2, if upon its service completion server 1 is free. Note that for exponential services this change of protocol can also be interpreted as if server 2 stops servicing a type- 1 job as long as server 1 is fre hereby kept busy longer, so that the loss probabilities are larger.

The modification guarantees JLB. This can be checked by substituting the probabilities given below in the job-local-balance equations (cf. [6] or [7]) and assuming exponential services. Roughly, it follows that also the "rate out of state $(0,1)$ due to the type-1 job at server 2 is made equal to 0 . By virtue of [6] and [7], the following stationary probabilities $p(\cdot)$ can now be concluded independently of the form of $G$ :

$\begin{array}{lcccccc}\text { Sutate } & (0,0) & (0,1) & (0,2) & (1,0) & (1,1) & (1,2) \\ p(\cdot) / \alpha & 1 & \rho & \rho & \rho & \rho^{2} & \rho^{2}\end{array}$

Table 1

Bounds and exact values for the simple grading

\begin{tabular}{|c|c|c|c|c|c|c|}
\hline \multirow[t]{2}{*}{$p$} & \multicolumn{3}{|l|}{ Type 1} & \multicolumn{3}{|l|}{ Type 2} \\
\hline & Lower & Exact & $\overline{\text { Upper }}$ & Lower & Exact & Upper \\
\hline 0.05 & 0.0012 & 0.003 & 0.004 & 0.048 & 0.050 & 0.090 \\
\hline 0.10 & 0.005 & 0.012 & 0.015 & 0.090 & 0.098 & 0.166 \\
\hline 0.50 & 0.076 & 0.152 & 0.166 & 0.333 & 0.394 & 0.500 \\
\hline 1.00 & 0.200 & 0.318 & 0.333 & 0.500 & 0.591 & 0.667 \\
\hline 2.50 & 0.471 & 0.587 & 0.595 & 0.714 & 0.804 & 0.833 \\
\hline 5.00 & 0.676 & 0.755 & 0.758 & 0.833 & 0.899 & 0.909 \\
\hline 7.50 & 0.768 & 0.826 & 0.827 & 0.882 & 0.932 & 0.938 \\
\hline 10.0 & 0.820 & 0.865 & C.866 & 0.909 & 0.949 & 0.952 \\
\hline 15.0 & 0.876 & 0.907 & 0.907 & 0.936 & 0.966 & 0.968 \\
\hline
\end{tabular}


where $\alpha$ denotes a normalizing constant. Since Poisson arrivals see time averages (cf. [11]), this leads to the upper bounds,

$B_{U}^{1}=2 p^{2} /\left[2 p^{2}+3 p+1\right] \geq B^{1}(G)$,

$B_{U}^{2}=\left[2 \rho^{2}+2 \rho\right] /\left[2 \rho^{2}+3 \rho+1\right] \geq B^{2}(G)$.

It has been shown in [10] that our modification corresponds to various possibilities of changing the exponential transition diagram, from which other interpretations can be extracted. Table 1 presents some numerical comparison with the exact values in the exponential case, as calculated by [9]. Throughout, the bounds reasonably estimate the order of magnitude of the exact values.

\section{A more complex overflow model and bounds}

This section extends the type-1 bounds of Section 2 by allowing more servers and different service speeds of regular and overflow servers. For simplicity of exposition, type 2 jobs are hereby omitted. More precisely, we consider a single Poisson arrival stream to a multi-server station with $c$ (regular) servers. The service speed of each of these servers is the same, without restriction of generality, say, equal to 1 . When all $c$ servers are busy, an arriving service request is rcrouted (overflowed) to a second station with $m$ (overflow) servers. The device speed of each of these is also assumed to be the same but possibly larger than 1 . Let $f$ denote this speed. Hence, $f \geq 1$. When both stations are saturated, an arriving service request is lost. Let $\lambda$ be the arrival rate and $G$ the distribution function of the service requirement (at both stations) with mean $\mu^{-1}$. We write $\rho_{1}=$ $(\lambda / \mu)$ and $\rho_{2}=(\lambda / \mu f)$ and are interested in the loss probability $B(G)$ of a service request.

Clearly, the present system is of practical importance. For $f=1$ it performes as the standard Erlang loss system with $c+m$ servers, so that Erlang's loss formula can be applied. For $f>1$, however, a number of servers is kept available which serve faster but which are put on (realistically at additional costs) only if the regular servers are congested. Since we need to differentiate between the number of jobs at stations 1 and 2, we can argue as in Section 2 that JLB fails. Correspondingly, a simple explicit formula is not available and insensitivity fails (cf. [6] and [7]).
To establish a lower bound $B_{L}$ of $B(G)$ independently of $G$, the following modification is suggested. Group the $c$ and $m$ servers together, where their service speeds are kept unchanged, and number them $1, \ldots, c, c+1, \ldots, c+m$. Further, let an arriving service request be randomly assigned to one of the free servers. That is, when $x$ servers are busy.a service request is assigned to any particular free server with probability $1 /(c+m-x)$. When all servers are busy it is lost. Intuitively, faster servers are hereby used more frequently than in the original system, so that the mean sojourn time of a job and consequently the loss probability will be reduced. Note that for $f=1$ our modification corresponds to the Erlang-bound for the type-1 jobs in the simple grading.

As in Section 2 for the upper bound, the modification guarantees JLB, which can be checked by substituting the probabilities given below in the job-local-balance equations (cf. [6] and [7]) and assuming exponential services. It can also be concluded from [6, Theorem 6.1]. The recognition of this modification, in fact, was based on the insight developed in the latter reference. In order to present the product-type stationary probabilities we need a detailed state description of the form $\left(i_{1}, i_{2}, \ldots, i_{n} ; j_{1}, j_{2}, \ldots, j_{k}\right)$ to denote that the servers $i_{1}, i_{2}, \ldots, i_{n}$ from the regular servers $1, \ldots, c$ and $j_{1}, j_{2}, \ldots, j_{k}$ from the faster servers $c+1, \ldots, c+m$ are busy. By virtue of [6] and [7], the following stationary probabilities $p(\cdot)$ can now be concluded independently of the form of G:

$$
\begin{aligned}
& p\left(i_{1}, i_{2}, \ldots, i_{n} ; j_{1}, j_{2}, \ldots, j_{k}\right) \\
& \quad=\alpha\left[\prod_{x=0}^{n+k-1} \frac{1}{(c+m-x)}\right]\left[\rho_{1}\right]^{n}\left[\rho_{2}\right]^{k},
\end{aligned}
$$

where $\alpha$ is a normalizing constant. Note that for $f=1$ and hence $\rho_{2}=\rho_{1}$, this formula coincides with Erlang's formula, since there are $\left(\begin{array}{c}c+m \\ n+k\end{array}\right)$ configurations with $(n+k)$ busy servers. Again, since Poisson arrivals see time averages (cf. [11]), a lower bound is thus established by

$B_{\mathrm{L}}=p(1,2, \ldots, c ; c+1, c+2, \ldots, c+m)$.

An upper bound can be found by the same type of modification as for the simple grading in Section 2. That is, let any job at one of the faster servers (at station 2) restart a complete new service at that server if upon its service completion all 
Table 2

Bounds for the oiverflow model $(\mu=1)$

\begin{tabular}{llllll}
\hline $\boldsymbol{c}$ & $\boldsymbol{m}$ & $\boldsymbol{\lambda}$ & $\boldsymbol{f}$ & $\boldsymbol{B}_{\mathbf{L}}$ & $\boldsymbol{B}_{\mathrm{U}}$ \\
\hline 3 & 1 & 6 & 2 & 0.395 & 0.443 \\
& & & 4 & 0.297 & 0.354 \\
& & 3 & 2 & 0.160 & 0.208 \\
& & & 4 & 0.104 & 0.148 \\
& & 2 & 2 & 0.074 & 0.105 \\
3 & 2 & 6 & 2 & 0.046 & 0.070 \\
& & 3 & 2 & 0.060 & 0.312 \\
5 & 1 & 6 & 2 & 0.211 & 0.270 \\
5 & & 3 & 2 & 0.037 & 0.066 \\
& 2 & 6 & 2 & 0.117 & 0.191 \\
& & 3 & 2 & 0.010 & 0.034 \\
\hline
\end{tabular}

regular servers (at station 1) are busy. As in Section 2, the following upper bound is then concluded.

$$
\begin{aligned}
& B_{U}=\left[\rho_{1}^{c} / c !\right]\left[\rho_{2}^{m} / m !\right] \\
& / \sum_{0 \leq n \leq c ; 0 \leq k \leq m}\left[\rho_{1}^{n} / n !\right]\left[\rho_{2}^{k} / k !\right] .
\end{aligned}
$$

Table 2 provides some numerical support by illustrating the width between the lower and upper bound in various situations. In the examples chosen the bounds are rather accurate. Clearly, however, their performance will become worse the larger the number of overflow servers. In any case, our results seem to indicate a usefulness to quick engineering.

\section{Evaluation}

An intuitively appealing bounding methodology is investigated for some simple overflow situations. The methodology is based on product-type modifications and secures insensitive quick bounds. Numerical suppori indicates a potential usefulness for quick engineering and seems promising for further investigation of the methodology.

\section{Acknowledgements}

I would like to thank Ad Ridder for indicating an error in an earlier version. I'm grateful also for the comments of an anonymous referee, which led to the analysis of Section 3.

\section{References}

[1] J.W. Cohen, "Sensitivity and insensitivity", Delft Progress Report 5, 159-173 (1980).

[2] N.M. van Dijk, "A(nother and general) proof of simple insensitive bounds for overflow systems", Research Report, Twenty Iniversity, Enschede, submitted to $A d v$. Appl. Prob. (1986).

[3] N.M. van Dijk and B.F. Lamond, "Bounds for the call congestion of finite single-server exponential tandem queues", Working Paper 1078, University of British Columbia, Vancouver, forthcoming in Operations Res. (1985).

[4] N.M. van Dijk and J. van der Wal, "Simple bounds for finite multi-server tandem queues", Research Report, Twente University, Enschede, submitted to Adv. Appl. Prob. (1986).

[5] N.M. van Dijk, J. Walrand anù P. Tsoucas, "Simple bounds for the call congestion of finite multi-server delay systems", Research Report, Twente University, Enschede, forthcoming in Probability in the Engineering and Informauonal Sciences, 1987.

[6] A. Hordijk and N.M. van Dijk, "Networks of queues, Part I: Job-local-balance and the adjoint process; Part II: General routine and service characteristics", Lecture Notes in Control and Information Sciences 60, 158-205 (1983).

[7] A. Hordijk and N.M. van Dijk, "Adjoint processes, joblocal-balance and insensitivity of stochastic networks", Bull. 44-th Session Int. Stat. Inst. 590, 776-788 (1983).

[8] A. Hordijk and A. Ridder, "Stochastic inequalities or an overflow model", Research Report, University of Leiden, Leiden, forthcoming in J. Appl. Prob. (1986).

[9] E.W.B. van Marion, "Influence of holding time distributions or blocking probabilities of a grading", TELE 20 , 17-20 (1968).

[10] A. Ridder, "A note on insensitive bounds for a grading", Research Report, University of Leiden, Leiden, 1986.

[11] R.W. Wolff, "Poisson arrivals see time averages", Operat. Res. 30, 223-231 (1982). 